\title{
A matroid associated with a phylogenetic tree
}

\author{
Andreas W.M. Dress $\|^{1} \quad$ Katharina T. Huber ${ }^{2} \quad$ Mike Steel $\|^{3 \|}$ \\ ${ }^{1}$ CAS-MPG Partner Institute and Key Lab for Computational Biology, Shanghai, China \\ ${ }^{2}$ School of Computing Sciences, University of East Anglia, Norwich, UK \\ ${ }^{3}$ Department of Mathematics and Statistics, University of Canterbury, Christchurch, New Zealand \\ received 19th Dec. 2013, revised 30 $0^{\text {th }}$ May 2014, accepted $2^{\text {nd }}$ June 2014.
}

A (pseudo-)metric $D$ on a finite set $X$ is said to be a 'tree metric' if there is a finite tree with leaf set $X$ and nonnegative edge weights so that, for all $x, y \in X, D(x, y)$ is the path distance in the tree between $x$ and $y$. It is well known that not every metric is a tree metric. However, when some such tree exists, one can always find one whose interior edges have strictly positive edge weights and that has no vertices of degree 2 , any such tree is - up to canonical isomorphism - uniquely determined by $D$, and one does not even need all of the distances in order to fully (re-)construct the tree's edge weights in this case. Thus, it seems of some interest to investigate which subsets of $\left(\begin{array}{c}X \\ 2\end{array}\right)$ suffice to determine ('lasso') these edge weights. In this paper, we use the results of a previous paper to discuss the structure of a matroid that can be associated with an (unweighted) $X$-tree $T$ defined by the requirement that its bases are exactly the 'tight edge-weight lassos' for $T$, i.e, the minimal subsets of $\left(\begin{array}{c}X \\ 2\end{array}\right)$ that lasso the edge weights of $T$.

Keywords: phylogenetic tree, tree metric, matroid, lasso (for a tree), cord (of a lasso)

\section{Introduction}

Given any finite tree $T$ without vertices of degree 2, there is an associated matroid $\mathbb{M}(T)$ having ground set $\left(\begin{array}{l}X \\ 2\end{array}\right)$ where $X$ is the set of leaves of $T$. In this paper, we describe this matroid and investigate a number of interesting properties it exhibits. The motivation for studying this matroid is its relevance to the problem of uniquely reconstructing an edge-weighted tree from its topology and just some of the leaf-to-leaf distances in that tree. This combinatorial problem arises in phylogenetics (the inference of evolutionary relationships from genetic data) since - due to patchy taxon coverage by available genetic loci (Sanderson et al. 2010) - reliable estimates of evolutionary distances can often be obtained only for some pairs of species.

In (Dress et al. 2011b), we already introduced and explored related mathematical questions. We asked when knowing just some of the leaf-to-leaf distances is sufficient to uniquely determine - or, as we say 'lasso' - the topology of the tree, or its edge weights, or both(i) In this paper, we turn our attention to a

\footnotetext{
*This work was partly supported by NSFC grant 11271255

${ }^{\dagger}$ Email: mike.steel@ canterbury.ac.nz

(i) Our term 'lasso', introduced in (Dress et al. 2011b), refers to the analogy of a cowboy catching an unruly beast (in our case a tree) with limited means. The term has no relationship to the use of the word in machine learning, or in linear regression.
} 
fixed (un-weighted) tree $T$ and the set of minimal subsets $\mathcal{L}$ of $\left(\begin{array}{c}X \\ 2\end{array}\right)$ for which the leaf-to-leaf distances between all $x, y \in X$ with $\{x, y\} \in \mathcal{L}$ relative to some edge-weighting $\omega$ of $T$ suffice to determine all the other distances relative to $\omega$ and, thus, the edge-weighting $\omega$. Indeed, these subsets form the bases of the matroid $\mathbb{M}(T)$ that will be studied here.

We begin by recalling some basic definitions and some relevant terminology from (Dress et al. 2011b) on trees, lassos, and associated concepts (readers unfamiliar with basic matroid theory may wish to consult (Welsh 1976) - though even Wikipedia may suffice). We then define $\mathbb{M}(T)$ and describe some of its basic properties before presenting our main results. Finally, we provide a number of remarks, observations, and questions for possible further study.

\section{Some terminology and basic facts}

We will assume throughout that $X$ is a finite set of cardinality $n \geq 3$ and, for any two elements $x, y \in X$, we will usually write just $x y$ instead of $\{x, y\}$, and we will refer to any such set as a 'cord' whenever $x \neq y$ holds. Throughout this paper, we will assume that $T=(V, E)$ is an $X$-tree, i.e., a finite tree with vertex set $V$, leaf set $X \subseteq V$, and edge set $E \subseteq\left(\begin{array}{c}V \\ 2\end{array}\right)$ that has no vertices of degree 2 . Two $X$-trees $T_{1}=\left(V_{1}, E_{1}\right)$ and $T_{2}=\left(V_{2}, E_{2}\right)$ are said to be 'equivalent' if there exists a bijection $\varphi: V_{1} \rightarrow V_{2}$ with $\varphi(x)=x$ for all $x \in X$ and $E_{2}$ coincides with $\left\{\{\varphi(u), \varphi(v)\}:\{u, v\} \in E_{1}\right\}$ in which case we will also write $T_{1} \simeq T_{2}$. In case every interior vertex of an $X$-tree $T$ (that is, every vertex in $V-X$ ) has degree 3 , $T$ will also be said to be a 'binary' $X$-tree.

Further, given any two vertices $u, v$ of $T$, we denote by $[u, v]$ the set of all vertices on the path in $T$ from $u$ to $v$ and by $E(u \mid v)=E_{T}(u \mid v)$ the set of all edges $e$ in $E$ on that path so that $[u, v]=\bigcup_{e \in E(u \mid v)} e$ always holds.

For each $e \in E$, we denote by $\omega_{e}$ the map $E \rightarrow \mathbb{R}: f \mapsto \delta_{e f}$ (where $\delta$ is, of course, the Kronecker delta function). And for all $e \in E$ and $x y \in\left(\begin{array}{c}X \\ 2\end{array}\right)$, we put $\delta_{e \mid x y}:=1$ in case $e \in E(x \mid y)$ and $\delta_{e \mid x y}:=0$ otherwise.

Here, given an $X$-tree $T=(V, E)$, we will be mainly concerned with the $\mathbb{R}$-linear map from $\mathbb{R}^{E}$ to $\mathbb{R}^{\left(\begin{array}{l}X \\ 2\end{array}\right)}$ defined by: $\omega \mapsto \omega^{T}$, where

$$
\omega^{T}(x y)=\sum_{e \in E(x \mid y)} \omega(e)=\sum_{e \in E} \omega(e) \delta_{e \mid x y},
$$

and the associated $\left(\begin{array}{l}X \\ 2\end{array}\right)$-labeled family of linear forms

$$
\lambda_{x y}=\lambda_{x y}^{T}: \mathbb{R}^{E} \rightarrow \mathbb{R}: \omega \mapsto \omega^{T}(x y) \quad\left(x y \in\left(\begin{array}{c}
X \\
2
\end{array}\right)\right) .
$$

Let $D_{\omega}=D_{(T, \omega)}$ denote the map from $X \times X$ to $\mathbb{R}_{\geq 0}$ associated to edge weighting $\omega$ and defined by

$$
(x, y) \mapsto D_{\omega}(x, y):=\sum_{e \in E(x \mid y)} \omega(e)
$$

Note that $\lambda_{x y}\left(\omega_{e}\right)=\delta_{e \mid x y}$ holds for all $e \in E$ and all $x, y \in X$, and that $D_{\omega}(x, y)=\lambda_{x y}(\omega)=$ $\omega^{T}(x y)$ for all $\omega \in \mathbb{R}^{E}$ and $x, y \in X$. 
When $\omega$ is a non-negative edge weighting, $D_{\omega}$ is nothing but the associated (pseudo-)metric on $X$ induced by the edge weighted tree $T=(T, \omega)$ and much studied in phylogenetic analysis.

Recall also that, given an arbitrary metric $D: X \times X \rightarrow \mathbb{R}_{\geq 0}$ defined on $X$,

- the metric $D$ is dubbed a 'tree metric' if it is of the form $D_{(T, \omega)}$ for some $X$-tree $T=(V, E)$ and some non-negative edge weighting $\omega: E \rightarrow \mathbb{R}_{\geq 0}$ of $T$

- which, in turn, holds if and only if $D$ satisfies the well-known 'four-point condition' stating that, for all $a, b, c, d$ in $X$, the larger two of the three distance sums $D(a, b)+D(c, d), D(a, c)+$ $D(b, d), D(a, d)+D(b, c)$ coincide,

- that, in this case, one can actually always find an $X$-tree $T$ and an edge weighting $\omega$ of $T$ with $D=D_{(T, \omega)}$ such that $\omega$ is strictly positive on all interior edges in which case $\omega$ is called a 'proper' edge weighting of $T$,

- any such pair $(T, \omega)$ is - up to canonical isomorphism - uniquely determined by $D$,

- and one does not even need to know the values of $D$ for all cords $x y$ in $\left(\begin{array}{c}X \\ 2\end{array}\right)$ in order to determine all the other distances and, thus, the edge-weighting $\omega$ in this case.

In this note, we continue our investigation of those subsets $\mathcal{L}$ of $\left(\begin{array}{c}X \\ 2\end{array}\right)$ for which - given the $X$-tree $T$ already the restriction $\left.\omega^{T}\right|_{\mathcal{L}}$ of the map $\omega^{T}$ to $\mathcal{L}$ suffices to determine - or 'lasso' - the edge weighting $\omega$ of $T$ that we began in (Dress et al. 2011b). To this end, we denote, for any subset $\mathcal{L}$ of $\left(\begin{array}{c}X \\ 2\end{array}\right)$,

- by $\langle\mathcal{L}\rangle=\langle\mathcal{L}\rangle^{T}$ the $\mathbb{R}$-linear subspace of the dual vector space $\widehat{\mathbb{R}}^{E}:=\operatorname{Hom}_{\mathbb{R}}\left(\mathbb{R}^{E}, \mathbb{R}\right)$ of the space $\mathbb{R}^{E}$ generated by the maps $\lambda_{x y}$ with $x y \in \mathcal{L}$,

- by $\operatorname{rk}(\mathcal{L})=\operatorname{rk}^{T}(\mathcal{L}):=\operatorname{dim}_{\mathbb{R}}\langle\mathcal{L}\rangle$ the dimension of $\langle\mathcal{L}\rangle$, and

- by $\Gamma(\mathcal{L}):=(X, \mathcal{L})$ the graph with vertex set $X$ and edge set $\mathcal{L}$.

Following the conventions introduced in (Dress et al., 2011b),

- we will refer to a subset $\mathcal{L}$ of $\left(\begin{array}{c}X \\ 2\end{array}\right)$ as being 'connected', 'disconnected' or 'bipartite' etc. whenever the graph $\Gamma(\mathcal{L})$ is connected, disconnected, or bipartite and so on,

- a connected component of $\Gamma(\mathcal{L})$ will also be called a connected component of $\mathcal{L}$,

- and given any two subsets $A, B$ of $X$, the subset $\{a b: a \in A, b \in B\}$ of $\left(\begin{array}{c}X \\ 2\end{array}\right)$ will be denoted by $A \vee B$ so that a subset $\mathcal{L}$ of $\left(\begin{array}{c}X \\ 2\end{array}\right)$ is bipartite if and only if there exist two disjoint subsets $A, B$ of $X$ with $\mathcal{L} \subseteq A \vee B$.

Further, a subset $\mathcal{L}$ of $\left(\begin{array}{c}X \\ 2\end{array}\right)$ will be called

- an 'edge-weight lasso' for $T$ if the implication " $\left.\omega_{1}^{T}\right|_{\mathcal{L}}=\left.\omega_{2}^{T}\right|_{\mathcal{L}} \Rightarrow \omega_{1}=\omega_{2}$ " holds for any two proper edge-weightings $\omega_{1}, \omega_{2}: E \rightarrow \mathbb{R}_{>0}$ of $T$,

- a 'topological lasso' for $T$ if the implication " $\left.\omega_{1}^{T^{\prime}}\right|_{\mathcal{L}}=\left.\omega_{2}^{T}\right|_{\mathcal{L}} \Rightarrow T^{\prime} \simeq T$ " holds for any $X$-tree $T^{\prime}$ and any proper edge-weightings $\omega_{1}$ of $T^{\prime}$ and $\omega_{2}$ of $T$, respectively, and

- a 'strong lasso' for $T$ if it is simultaneously an edge-weight and a topological lasso for $T$.

Next, recall (see e.g. (Oxley, 2011; Welsh, 1976)) that an 'abstract' matroid $\mathbb{M}$ with a ground set, say, $M$ can be defined in terms of its 'rank function' $\mathrm{rk}_{\mathbb{M}}: \mathcal{P}(M) \rightarrow \mathbb{N}_{0}$ (and with $\mathcal{P}(M)$ denoting the power set of $M)$ as well as by the collection $\mathbb{I}_{\mathbb{M}}=\left\{I \subseteq M: \operatorname{rk}_{\mathbb{M}}(I)=|I|\right\}$ of its 'independent sets', the collection $\mathbb{G}_{\mathbb{M}}=\left\{L \subseteq M: \operatorname{rk}_{\mathbb{M}}(L)=\operatorname{rk}_{\mathbb{M}}(M)\right\}$ of its 'generating sets', the collection $\mathbb{B}_{\mathbb{M}}$ of its 'bases', 
i.e., the maximal sets in $\mathbb{I}_{\mathbb{M}}$ or, just as well, the minimal sets in $\mathbb{G}_{\mathbb{M}}$, the collection $\mathbb{C}_{\mathbb{M}}$ of its 'circuits', i.e., the minimal sets in $\mathcal{P}(M)-\mathbb{I}_{\mathbb{M}}$, as well as the 'closure operator' $[\ldots]_{\mathbb{M}}: \mathcal{P}(M) \rightarrow \mathcal{P}(M): L \mapsto[L]_{\mathbb{M}}:=$ $\left\{m \in M: \operatorname{rk}_{\mathbb{M}}(L)=\operatorname{rk}_{\mathbb{M}}(L \cup\{m\})\right\}$ associated to $\mathbb{M}$.

Here, given any $X$-tree $T$, we want to investigate the matroid $\mathbb{M}(T)$ with ground set $M:=\left(\begin{array}{l}X \\ 2\end{array}\right)$ associated to $T$ whose rank function $\operatorname{rk}_{\mathbb{M}(T)}$ is the map $\operatorname{rk}^{T}: \mathcal{P}\left(\left(\begin{array}{l}X \\ 2\end{array}\right)\right) \rightarrow \mathbb{N}_{0}$ defined just above, i.e., the matroid that is 'represented' (over $\mathbb{R}$, again see e.g. (Oxley, 2011; Welsh, 1976) ) by the map

$$
\lambda^{T}:\left(\begin{array}{c}
X \\
2
\end{array}\right) \rightarrow \widehat{\mathbb{R}^{E}}: x y \mapsto \lambda_{x y}
$$

We will denote by $\mathbb{I}(T):=\mathbb{I}_{\mathbb{M}(T)}$ its collection of independent sets, by $\mathbb{G}(T):=\mathbb{G}_{\mathbb{M}(T)}$ its collection of generating sets, by $\mathbb{B}(T):=\mathbb{B}_{\mathbb{M}(T)}$ its collection of bases, by $\mathbb{C}(T):=\mathbb{C}_{\mathbb{M}(T)}$ its collection of 'circuits' and, given any subset $\mathcal{L}$ of $\left(\begin{array}{c}X \\ 2\end{array}\right)$, we denote by $[\mathcal{L}]^{T}:=[\mathcal{L}]_{\mathbb{M}(T)}$ the '(T-)closure' of $\mathcal{L}$ relative to $\mathbb{M}(T)$.

An alternative description of $\mathbb{M}(T)$ is as the matroid associated with the $(0,1)$ matrix $A(T)=\left[A_{(e, x y)}\right]$, where the row index $e$ ranges over the set $E$ of edges of $T$, and the column index $x y$ ranges over $\left(\begin{array}{c}X \\ 2\end{array}\right)$, with:

$$
A_{(e, x y)}:= \begin{cases}1, & \text { if } e \in E(x \mid y) \\ 0, & \text { otherwise }\end{cases}
$$

By (Dress et al. 2011b. Theorem 1), it follows that for any subset $\mathcal{L}$ of $\left(\begin{array}{c}X \\ 2\end{array}\right)$ the following are equivalent.

(a) $\mathcal{L}$ is an edge-weight lasso for an $X$-tree $T=(V, E)$;

(b) the implication " $\left.\omega_{1}^{T}\right|_{\mathcal{L}}=\left.\omega_{2}^{T}\right|_{\mathcal{L}} \Rightarrow \omega_{1}=\omega_{2}$ " holds not only for any two proper edge weightings $\omega_{1}, \omega_{2}$ of $T$, but for any two maps $\omega_{1}, \omega_{2} \in \mathbb{R}^{E}$;

(c) $\langle\mathcal{L}\rangle$ coincides with $\widehat{\mathbb{R}^{E}}$;

(d) $\mathcal{L} \in \mathbb{G}(T)$, or, equivalently, $\operatorname{rk}^{T}(\mathcal{L})=\operatorname{rk}^{T}\left(\left(\begin{array}{l}X \\ 2\end{array}\right)\right)=|E|$ holds.

In particular, an edge-weight lasso $\mathcal{L}$ for $T$ is a 'tight' edge-weight lasso for $T$, i.e, a minimal subset of $\left(\begin{array}{c}X \\ 2\end{array}\right)$ that is an edge-weight lasso for $T$, if and only if its cardinality coincides with $|E|$ if and only if it is a basis of $\mathbb{M}(T)$, that is, $\mathcal{L} \in \mathbb{B}(T)$ holds.

Particular types of $X$-trees that will play an important role in this paper are shown in Figure 1 . They comprise (i) the 'star trees', i.e., $X$-trees that have just one interior vertex and, hence, are equivalent to the tree $T^{\star}(X):=\left(V^{\star}(X), E^{\star}(X)\right)$ with leaf set $X$, vertex set $V^{\star}(X):=X \dot{\cup}\{\star\}$ and edge set $E^{\star}(X):=\{\star x: x \in X\}$ where ' $\star$ ' denotes just some arbitrary, but fixed element not in $X$; (ii) 'quartet trees', i.e., binary $X$-trees that have four leaves (with $T_{a b \mid c d}$ denoting the quartet tree with leaf set $\{a, b, c, d\}$ whose central edge that will also be denoted by $e_{a b \mid c d}$ separates the leaves $a, b$ from $\left.c, d\right)$, and (iii) 'caterpillar trees', i.e. binary $X$-trees $T=(V, E)$ containing two interior vertices $u, v \in V-X$ with $[u, v]=V-X$. 


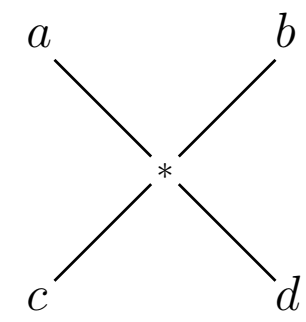

(i)

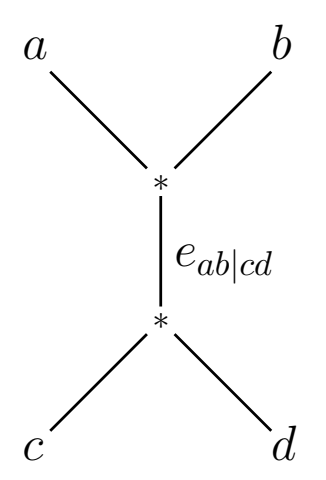

(ii)

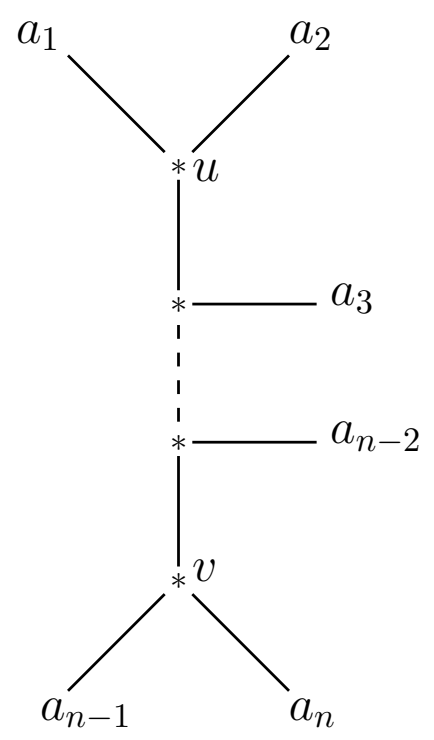

(iii)

Fig. 1:

(i) A star tree with leaf set $X_{4}:=\{a, b, c, d\}$; (ii) A binary $X_{4}$-tree - up to equivalence, there are two more binary $X_{4}$-trees ; (iii) a 'caterpillar' $X_{n}$-tree for $X_{n}:=\left\{a_{1}, a_{2}, \ldots, a_{n-1}, a_{n}\right\}$.

\section{Star trees}

We first consider the simplest type of $X$-tree, i.e., the star tree $T^{\star}:=T^{\star}(X)$ with leaf set $X$ (cf. Figure1 (i)), for which the associated $(0,1)$ matrix $A(T)$ has just two nonzero entries in each column. In this case, the associated matroid $\mathbb{B}\left(T^{\star}\right)$ is well known: It is easily seen to exactly coincide with the 'biased matroid' of the complete signed graph $\left(X,\left(\begin{array}{c}X \\ 2\end{array}\right)\right)$ with vertex set $X$ all of whose edges have sign -1 . In consequence (see e.g. (Oxley, 2011, Section 6.10) and the references therein to Zaslavsky's papers on signed graphic matroids), the following results are known to hold:

Proposition 3.1 Given a finite set $X$ of cardinality $n \geq 3$, the following holds for the matroid $\mathbb{B}\left(T^{\star}\right)$ associated to the star tree $T^{\star}$ with leaf set $X$ :

(i) The collection $\mathbb{G}\left(T^{\star}\right)$ of all edge-weight lassos for $T^{\star}$ coincides with the collection of all 'strongly non-bipartite' subsets $\mathcal{L}$ of $\left(\begin{array}{c}X \\ 2\end{array}\right)$, i.e, all subsets $\mathcal{L}$ of $\left(\begin{array}{c}X \\ 2\end{array}\right)$ for which none of the connected components of $\mathcal{L}$ is bipartite.

(ii) The collection $\mathbb{B}\left(T^{\star}\right)$ of all tight edge-weight lassos for $T^{\star}$ coincides with the collection of all minimal strongly non-bipartite subsets $\mathcal{L}$ of $\left(\begin{array}{c}X \\ 2\end{array}\right)$, i.e, all subsets $\mathcal{L}$ of $\left(\begin{array}{c}X \\ 2\end{array}\right)$ for which each connected component of $\mathcal{L}$ contains exactly one circle ${ }^{(i i)}$ and the length of this circle has odd parity.

\footnotetext{
(ii) In our context, we adopt the convention of calling a graph (and, hence, also every subgraph of a graph) a 'circle' if it is connected and every vertex in that graph has degree 2 .
} 
(iii) The collection $\mathbb{I}\left(T^{\star}\right)$ of all independent subsets of $\mathbb{M}\left(T^{\star}\right)$ coincides with the collection of all subsets $\mathcal{L}$ of $\left(\begin{array}{c}X \\ 2\end{array}\right)$ for which each connected component of $\mathcal{L}$ is either a tree or contains exactly one circle and the length of this circle has odd parity.

(iv) The collection $\mathbb{C}\left(T^{\star}\right)$ of all circuits of $\mathbb{M}\left(T^{\star}\right)$ coincides with the collection of all subsets $\mathcal{L}$ of $\left(\begin{array}{c}X \\ 2\end{array}\right)$ that either form a circle of even length or a pair of circles of odd length together with a connecting simple path, such that the two circles are either disjoint (then the connecting path has one end in common with each circle and is otherwise disjoint from both) or share just a single common vertex (in this case the connecting path is that single vertex).

(v) The co-rank $n-\mathrm{rk}^{T^{\star}}(\mathcal{L})$ of a subset $\mathcal{L}$ of $\left(\begin{array}{c}X \\ 2\end{array}\right)$ relative to $\mathbb{M}\left(T^{\star}\right)$ coincides with the number of bipartite connected component of $\mathcal{L}$.

(vi) The closure $[\mathcal{L}]^{T^{\star}}$ of a subset $\mathcal{L}$ of $\left(\begin{array}{c}X \\ 2\end{array}\right)$ relative to $\mathbb{M}\left(T^{\star}\right)$ coincides with the union of (a) the edge set of the complete graph whose vertex set is the union of the vertex sets of all non-bipartite connected components of $\mathcal{L}$ and (b) all subsets of the form $A \vee B$ for which some bipartite connected component $\mathcal{L}^{\prime}$ of $\mathcal{L}$ with $\mathcal{L}^{\prime} \subseteq A \vee B, A \cup B=\bigcup_{x y \in \mathcal{L}^{\prime}}\{x, y\}$, and $A \cap B=\emptyset$ exists.

\section{A recursive approach for computing $\mathbb{B}(T)$}

Every $X$-tree can be reduced by a sequence of edge contractions to a star tree (one may even insist that at each stage, one of the two subtrees incident with the edge being contracted has only one non-leaf vertex, though we do not require this here). Thus, Proposition 3.1 can be used as basis for a recursive description of the matroid associated with any $X$-tree, provided that one can describe, for any $X$-tree $T$, how to obtain $\mathbb{B}(T)$ from $\mathbb{B}(T / f)$ where $f$ is any interior edge of $T$, and $T / f$ is the $X$-tree obtained from $T$ by collapsing edge $f$. We provide such a description shortly, in Proposition 4.2 , using the following lemma the statement of which relies on further terminology which we introduce next. Suppose $T=(V, E)$ is an $X$-tree and $F$ is a subset of the set of interior edges of $T$. Then we denote by $T / F$ the $X$-tree obtained by collapsing all edges in $F$. Furthermore, we denote by $\left.\lambda\right|_{E-F}$ the restriction of a map $\lambda \in \widehat{\mathbb{R}^{E}}$ to the space $\mathbb{R}^{E-F}$ relative to the canonical embedding $\mathbb{R}^{E-F} \rightarrow \mathbb{R}^{E}: \omega \mapsto \omega_{(F \rightarrow 0)}$ defined by extending each map $\omega \in \mathbb{R}^{E-F}$ to the map $\omega_{(F \rightarrow 0)}$ by putting $\omega_{(F \rightarrow 0)}(e):=0$ for all $e \in F$.

Lemma 4.1 Given any $X$-tree $T=(V, E)$, any subset $F$ of the set of interior edges of $T$, any map $\lambda \in \widehat{\mathbb{R}^{E}}$, and any map $\rho:\left(\begin{array}{c}X \\ 2\end{array}\right) \rightarrow \mathbb{R}$ with $\lambda=\sum_{x y \in\left(\begin{array}{l}X \\ 2\end{array}\right)} \rho(x y) \lambda_{x y}^{T}$. Then,

$$
\left.\lambda\right|_{E-F}=\sum_{x y \in\left(\begin{array}{l}
X \\
2
\end{array}\right)} \rho(x y) \lambda_{x y}^{T / F},
$$

i.e., one has $\lambda(\omega)=\sum_{x y \in\left(\begin{array}{c}X \\ 2\end{array}\right)} \rho(x y) \lambda_{x y}^{T / F}\left(\left.\omega\right|_{E-F}\right)$ for all maps $\omega \in \mathbb{R}^{E}$ with $\omega(f)=0$ for all $f \in F$.

In particular, if $\mathcal{L}$ is a edge-weight lasso for $T$, then $\mathcal{L}$ is also an edge-weight lasso for the $X$-tree $T / F$. More generally,

$$
\operatorname{rk}^{T}(\mathcal{L})=\operatorname{rk}^{T / F}(\mathcal{L})+\operatorname{dim}\left\{\lambda \in\langle\mathcal{L}\rangle^{T}: \lambda(e)=0 \text { for all } e \in E-F\right\} \leq \mathrm{rk}^{T / F}(\mathcal{L})+|F|
$$

holds for every subset $\mathcal{L}$ of $\left(\begin{array}{c}X \\ 2\end{array}\right)$ and any subset $F$ of the set of interior edges of $T$. 
Proof: The first part follows directly from the definitions and implies that $\lambda_{x y}^{T / F}=\left.\lambda_{x y}^{T}\right|_{E-F}$ holds for all $x y \in\left(\begin{array}{c}X \\ 2\end{array}\right)$. In particular, as the map $\widehat{\mathbb{R}^{E}} \rightarrow \widehat{\mathbb{R}^{E-F}}:\left.\lambda \mapsto \lambda\right|_{E-F}$ is surjective, the maps $\lambda_{x y}^{T / F}\left(x y \in\left(\begin{array}{c}X \\ 2\end{array}\right)\right)$ must generate $\widehat{\mathbb{R}^{E-F}}$ whenever the maps $\lambda_{x y}^{T}\left(x y \in\left(\begin{array}{c}X \\ 2\end{array}\right)\right)$ generate $\widehat{\mathbb{R}^{E}}$ while, more generally, they generate a space whose dimension coincides with the difference of $\operatorname{rk}^{T}(\mathcal{L})$ and the dimension of the kernel of the map $\langle\mathcal{L}\rangle^{T} \rightarrow \widehat{\mathbb{R}^{E-F}}:\left.\lambda \mapsto \lambda\right|_{E-F}$.

Proposition 4.2 Given an $X$-tree $T$, an interior edge $f$ of $T$, a pair $x y \in\left(\begin{array}{c}X \\ 2\end{array}\right)$, and a basis $B$ of $\mathbb{M}(T / f)$, let $\rho_{x y} \in \mathbb{R}^{B}$ denote the unique map in $\mathbb{R}^{B}$ with $\lambda_{x y}^{T / f}=\sum_{b \in B} \rho_{x y}(b) \lambda_{b}^{T / f}$. Then, $\mathbb{B}(T)$ coincides with the set

$$
\mathbb{B}_{f}:=\left\{\{x y\} \cup B: x y \in\left(\begin{array}{c}
X \\
2
\end{array}\right), B \in \mathbb{B}(T / f), \text { and } \sum_{b \in B} \rho_{x y}(b) \delta_{f b} \neq \delta_{f \mid x y}\right\} .
$$

Proof: By Lemma 4.1, there exists, for each $B^{\prime} \in \mathbb{B}(T)$, some $b^{\prime} \in B^{\prime}$ with $B^{\prime}-b^{\prime} \in \mathbb{B}(T / f)$. Thus, each element of $\mathbb{B}(T)$ is of the form $B \cup\{x y\}$ for some $x y \in\left(\begin{array}{c}X \\ 2\end{array}\right)$ and some $B \in \mathbb{B}(T / f)$. So, $\mathbb{B}(T) \subseteq\left\{\{x y\} \cup B: x y \in\left(\begin{array}{c}X \\ 2\end{array}\right), B \in \mathbb{B}(T / f)\right\}$ must clearly hold. Now suppose that $x y \in\left(\begin{array}{c}X \\ 2\end{array}\right)$ and $B \in \mathbb{B}(T / f)$ holds. Then, denoting by $\Lambda_{B, x y}$ the space of all maps $\lambda \in\langle\{x y\} \cup B\rangle^{T}$ with $\left.\lambda\right|_{E-\{f\}}=0$, it follows from $\sqrt{1}$ that $\{x y\} \cup B \in \mathbb{B}(T) \Longleftrightarrow \operatorname{dim} \Lambda_{B, x y}=1$ holds while, by construction, we have $\operatorname{dim} \Lambda_{B, x y}=1$ if and only if there exists some non-zero map in $\Lambda_{B, x y}$.

However, given any map $\rho \in \mathbb{R}^{B}$ and any real number $c$, it follows from the fact that, by definition, $\left.\lambda_{z z^{\prime}}^{T}\right|_{E-\{f\}}$ coincides with $\lambda_{z z^{\prime}}^{T / f}$ for all $z z^{\prime} \in\left(\begin{array}{c}X \\ 2\end{array}\right)$, one has $\lambda_{c, \rho}:=-c \lambda_{x y}^{T}+\sum_{b \in B} \rho(b) \lambda_{b}^{T} \in \Lambda_{B, x y}$ if and only if $-c \lambda_{x y}^{T / f}+\sum_{b \in B} \rho(b) \lambda_{b}^{T / f}$ vanishes and, hence, if and only if $\rho(b)=c \rho_{x y}(b)$ holds for all $b \in B$. Thus, one has $\{x y\} \cup B \in \mathbb{B}(T)$ if and only if one has $\lambda_{c, \rho}\left(\omega_{f}\right) \neq 0$ and, hence, if and only if $\sum_{b \in B} \rho_{x y}(b) \delta_{f b} \neq \delta_{f \mid x y}$ holds as claimed.

Remark: Suppose that $T=(V, E)$ is an $X$-tree and that $U \subseteq V$ is a $T$-core as defined in (Dress et al. 2011b. Section 5), i.e., a non-empty subset of $V$ for which the induced subgraph $T_{U}:=\left(U, E_{U}:=\right.$ $\{e \in E: e \subseteq U\}$ ) of $T$ with vertex set $U$ is connected (and, hence, a tree) and the degree $\operatorname{deg}_{T_{U}}(v) \operatorname{lof}$ any vertex $v$ in $T_{U}$ is either 1 or coincides with the degree $\operatorname{deg}_{T}(v)$ of $v$ in $T$. Then, the $\operatorname{rank}^{T}(\mathcal{L})$ of a subset $\mathcal{L}$ of $\left(\begin{array}{c}X \\ 2\end{array}\right)$ relative to $T$ and the rank $\operatorname{rk}^{T_{U}}\left(\mathcal{L}_{U}\right)$ of the corresponding subset $\mathcal{L}_{U}$ of $\left(\begin{array}{c}X_{U} \\ 2\end{array}\right)$ relative to the $X_{U}$-tree $T_{U}$ are easily seen to be related by the inequality

$$
\mathrm{rk}^{T}(\mathcal{L}) \leq \operatorname{rk}^{T_{U}}\left(\mathcal{L}_{U}\right)+\left|E-E_{U}\right|
$$

This fact can be used to prove (Dress et al., 2011b, Theorem 5) in the same way as Lemma 4.1 has been used above to establish Proposition 4.2

\subsection{An example}

To illustrate Proposition 4.2 consider - for $X:=\{a, b, c, d\}$ - the quartet $X$-tree $T:=T_{a b \mid c d}$ shown in Figure 1(ii). In this case, there is - up to scaling - only one linear relation between the six maps $\lambda_{x y}^{T}\left(x y \in\left(\begin{array}{c}X \\ 2\end{array}\right)\right)$, viz. the relation

$$
\lambda_{a c}^{T}+\lambda_{b d}^{T}=\lambda_{a d}^{T}+\lambda_{b c}^{T}: E^{\mathbb{R}} \rightarrow \mathbb{R}: \omega \mapsto 2 \omega\left(e_{a b \mid c d}\right)+\sum_{e \in E, e \neq e_{a b \mid c d}} \omega(e) .
$$


Thus, $\mathbb{B}(T)$ consists of the four 5 -subsets $\mathcal{L}$ of $\left(\begin{array}{c}X \\ 2\end{array}\right)$ that do not contain exactly one of the four cords $a c, a d, b c, b d$ - or, equivalently, with $|\mathcal{L} \cap\{a c, a d, b c, b d\}|=3$ - and, hence, the four subsets $\mathcal{L}$ of $\left(\begin{array}{l}X \\ 2\end{array}\right)$ whose graphs $\Gamma(\mathcal{L})$ are shown in Figure 2 (ii). Clearly, if $f$ coincides with the unique interior edge of $T_{a b \mid c d}$ (i.e., the edge denoted by $e_{a b \mid c d}$ in Figure 1 (ii)), $T / f$ is equivalent to the star tree $T^{\star}:=T^{\star}(X)$, also shown in Figure 1 (i). Furthermore, the graphs $\Gamma(\mathcal{L})$ corresponding to the bases $\mathcal{L}$ in $\mathbb{B}\left(T^{\star}\right)$, being minimal strongly non-bipartite graphs with vertex set $X$, must consist of one triangle (for which there are 4 possibilities) to which the remaining element in $X$ is appended by a single edge (for which there are 3 possibilities). So, $\mathbb{B}\left(T^{\star}\right)$ consists of 12 bases that form two orbits relative to the symmetry group of $T$ representatives of which are the bases $B_{1}:=\{a b, b c, c a, d a\}$ and $B_{2}:=\{a b, b c, c a, d c\}$ shown in Figure 2(i). For the two cords $d b, d c \in\left(\begin{array}{c}X \\ 2\end{array}\right)-B_{1}$, we have - putting $f:=e_{a b \mid c d}-$

$$
\lambda_{d b}^{T / f}=\lambda_{d a}^{T / f}-\lambda_{a c}^{T / f}+\lambda_{c b}^{T / f} \quad \text { and } \quad \lambda_{d c}^{T / f}=\lambda_{d a}^{T / f}-\lambda_{a b}^{T / f}+\lambda_{b c}^{T / f}
$$

while

$$
\lambda_{d b}^{T}\left(\omega_{f}\right)=\lambda_{d a}^{T}\left(\omega_{f}\right)-\lambda_{a c}^{T}\left(\omega_{f}\right)+\lambda_{c b}^{T}\left(\omega_{f}\right)=1
$$

and

$$
\lambda_{d c}^{T}\left(\omega_{f}\right)=0 \neq \lambda_{d a}^{T}\left(\omega_{f}\right)-\lambda_{a b}^{T}\left(\omega_{f}\right)+\lambda_{b c}^{T}\left(\omega_{f}\right)=2
$$

holds implying that, to bases of type $B_{1}$, we can add cords of type $d c$, but not cords of type $d b$.

And for the two cords $d a, d b \in\left(\begin{array}{c}X \\ 2\end{array}\right)-B_{2}$, we have

$$
\lambda_{d a}^{T / f}=\lambda_{d c}^{T / f}-\lambda_{c b}^{T / f}+\lambda_{b a}^{T / f} \quad \text { and } \quad \lambda_{d b}^{T / f}=\lambda_{d c}^{T / f}-\lambda_{c a}^{T / f}+\lambda_{a b}^{T / f}
$$

as well as

$$
1=\lambda_{d a}^{T}\left(\omega_{f}\right) \neq \lambda_{d c}^{T}\left(\omega_{f}\right)-\lambda_{c b}^{T}\left(\omega_{f}\right)+\lambda_{b a}^{T}\left(\omega_{f}\right)=-1
$$

and

$$
1=\lambda_{d b}^{T}\left(\omega_{f}\right) \neq \lambda_{d c}^{T}\left(\omega_{f}\right)-\lambda_{c a}^{T}\left(\omega_{f}\right)+\lambda_{a b}^{T}\left(\omega_{f}\right)=-1
$$

holds implying that, to bases of type $B_{2}$, we can add either one of the two missing cords. Obviously, this fully corroborates our previous assertion about $\mathbb{B}\left(T_{a b \mid c d}\right)$.

Our next results require two definitions that will also be important later in this paper: Recall first that, given an $X$-tree $T$ and a subset $Y$ of $X$ of cardinality at least 3, one denotes

- by $\left.T\right|_{Y}$ the $Y$-tree obtained from the minimal subtree of $T$ that connects the leaves in $Y$ by suppressing any resulting vertices of degree 2 (see e.g. (Dress et al. 2011b, Section 2.3)),

- by $\left.V\right|_{Y}$ and $\left.E\right|_{Y}$ its vertex and edge set, respectively,

- and, given in addition any edge weighting $\omega$ of $T$, one denotes by $\left.\omega\right|_{Y}$ the 'induced' edge weighting of $\left.T\right|_{Y}$, i.e., the edge weighting that maps any edge $\left.\{u, v\} \in E\right|_{Y}$ onto the sum $\sum_{e \in E(u \mid v)} \omega(e)$, yielding a surjective $\mathbb{R}$-linear map res $\operatorname{res}_{Y}: \mathbb{R}^{E} \rightarrow \mathbb{R}^{\left.E\right|_{Y}}:\left.\omega \mapsto \omega\right|_{Y}$ such that $\lambda_{y y^{\prime}}^{T}(\omega)=\omega^{T}\left(y y^{\prime}\right)=$ $\left(\left.\omega\right|_{Y}\right)^{\left.T\right|_{Y}}\left(y y^{\prime}\right)=\lambda_{y y^{\prime}}^{\left.T\right|_{Y}}\left(\operatorname{res}_{Y}(\omega)\right)$ holds for all $\omega \in \mathbb{R}^{E}$ and all $y y^{\prime} \in\left(\begin{array}{c}Y \\ 2\end{array}\right)$. 

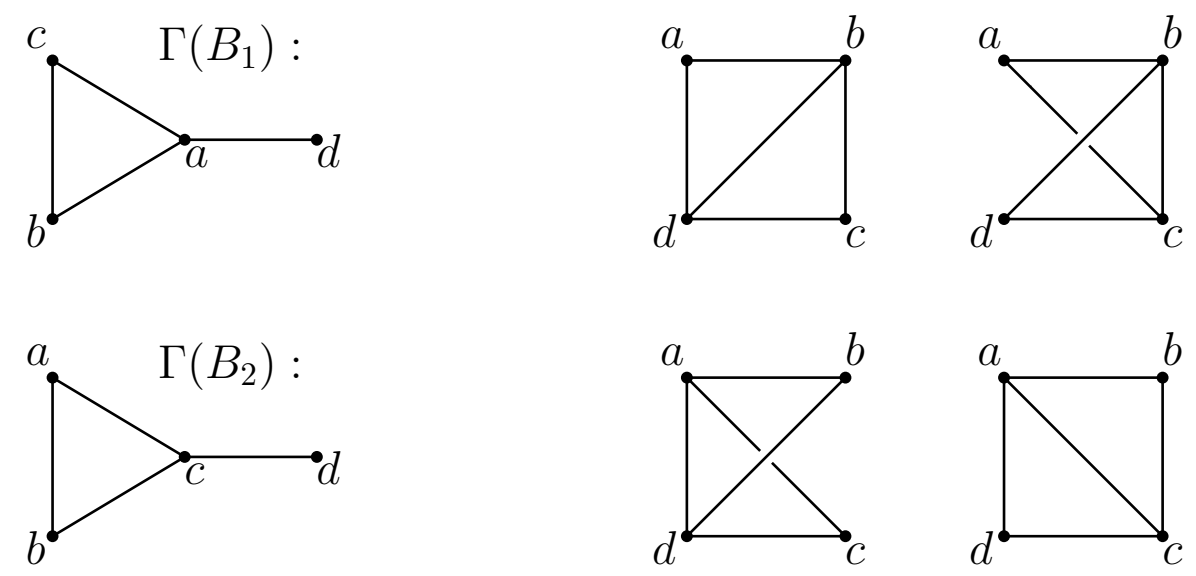

Fig. 2:

(i): Two graphs representing two of the twelve tight edge-weight lassos of $T^{\star}(\{a, b, c, d\})$, one from each of the two orbits of such lassos relative to the 8-element symmetry group of $T_{a b \mid c d}$.

(ii): The four graphs associated to the four bases in $\mathbb{B}\left(T_{a b \mid c d}\right)$.

It follows that the map $\lambda_{y y^{\prime}}^{T}: \mathbb{R}^{E} \rightarrow \mathbb{R}$ coincides, for all $y y^{\prime} \in\left(\begin{array}{c}Y \\ 2\end{array}\right)$, with the map $\lambda_{y y^{\prime}}^{\left.T\right|_{Y}} \circ \operatorname{res}_{Y}$, the composition of the maps res $\operatorname{ran}_{Y}$ and $\lambda_{y y^{\prime}}^{\left.T\right|_{Y}}$.

So, denoting by $\widehat{\text { resYY }}$ the dual - and necessarily injective - map $\widehat{\mathbb{R}^{\left.E\right|_{Y}}} \rightarrow \widehat{\mathbb{R}^{E}}: \lambda \mapsto \lambda \circ \operatorname{res}_{Y}$ of the map $\operatorname{res}_{Y}$, we have also $\langle\mathcal{L}\rangle^{T}=\widehat{\operatorname{res}_{Y}}\left(\langle\mathcal{L}\rangle^{\left.T\right|_{Y}}\right)$ and $\operatorname{rk}^{T}(\mathcal{L})=\operatorname{rk}^{\left.T\right|_{Y}}(\mathcal{L})$ for every subset $\mathcal{L}$ of $\left(\begin{array}{c}Y \\ 2\end{array}\right)$. In consequence, we must also have

$$
\mathrm{rk}^{T}(\mathcal{L})=\operatorname{rk}^{\left.T\right|_{Y}}(\mathcal{L})
$$

for every subset $Y$ of $X$ of cardinality at least 3 and every subset $\mathcal{L}$ of $\left(\begin{array}{c}Y \\ 2\end{array}\right)$. Consequently, every circuit $\mathcal{L} \subseteq\left(\begin{array}{c}Y \\ 2\end{array}\right)$ of $\mathbb{M}\left(\left.T\right|_{Y}\right)$ must also be a circuit of $\mathbb{M}(T)$, i.e., we have $\mathbb{C}\left(\left.T\right|_{Y}\right) \subseteq \mathbb{C}(T)$ for every such subset $Y$ of $X$ of cardinality at least 3 .

Further, denoting - for every $x \in X$ - by $e_{x} \in E$ the unique pendant edge of $T$ containing $x$, we say that a cord $a b$ of $X$ forms (or 'is') a ' $T$-cherry' if the two edges $e_{a}, e_{b} \in E$ share a vertex, and $a b$ is said to form a 'proper $T$-cherry' if this vertex has degree 3. Note that a cord $a b$ of $X$ forms a proper $T$-cherry if and only if $\left.T\right|_{\{a, b, c, d\}} \simeq T_{a b \mid c d}$ holds for any two distinct elements $c, d$ in $X-\{a, b\}$ (if any). Note also that, in a binary $X$-tree $T$, every $T$-cherry is a proper $T$-cherry. In addition, such a tree is a caterpillar tree if and only if $|X|=3$ holds or its leaf set contains exactly two distinct $T$-cherries. We claim:

Proposition 4.3 For an $X$-tree $T$, a cord $a b \in\left(\begin{array}{c}X \\ 2\end{array}\right)$ is a 'co-loop' of $\mathbb{M}(T)$, i.e. it is contained in every edge-weight lasso for $T$, if and only if ab is a proper $T$-cherry. 
Proof: If $|X|=3$ holds, the set $\left(\begin{array}{c}X \\ 2\end{array}\right)$ is the only basis of $\mathbb{M}(T)$ while, if $n \geq 4$ holds and $a b$ is a proper $T$-cherry, the cord $a b$ must be contained in every edge-weight lasso for $T$ in view of (Dress et al. 2011b. Corollary 1). Conversely, if $a b$ does not form a proper $T$-cherry, there must exist two distinct elements $c, d$ in $X-\{a, b\}$ such that $\lambda_{a b}^{T}+\lambda_{c d}^{T}=\lambda_{a c}^{T}+\lambda_{b d}^{T}$ holds. This implies that $\lambda_{a b}^{T}$ cannot be a co-loop of $\mathbb{M}(T)$.

\section{Main results}

\section{1 $\mathbb{M}(T)$ determines $T$ up to equivalence}

We begin this section by showing that the matroid associated with an $X$-tree determines that $X$-tree, up to equivalence:

Theorem 1 One has " $\mathbb{M}\left(T_{1}\right)=\mathbb{M}\left(T_{2}\right) \Longleftrightarrow T_{1} \simeq T_{2}$ " for any two $X$-trees $T_{1}$ and $T_{2}$.

Proof: We first note that, if $T$ is any $X$-tree and $Y=\{a, b, c, d\}$ is a 4-subset of $X$, then we have $\left.T\right|_{Y} \simeq$ $T_{a b \mid c d}$ if and only if there exists at least one basis $B$ of $\mathbb{M}(T)$ containing the set $\mathcal{L}_{a b c d}:=\{a b, b c, c d, d a\}$ : Indeed, if $\left.T\right|_{Y} \simeq T_{a b \mid c d}$ holds, the four maps $\lambda_{x y}^{\left.T\right|_{Y}}\left(x y \in \mathcal{L}_{a b c d}\right)$ and, hence, also the corresponding four maps $\lambda_{x y}^{T}\left(x y \in \mathcal{L}_{a b c d}\right)$ are linearly independent. So, by the matroid augmentation property of independent sets, there exists some $B \in \mathbb{M}(T)$ containing these four cords. Conversely, if $\left.T\right|_{Y} \nsucceq T_{a b \mid c d}$ and, therefore, also $\lambda_{a b}^{T}+\lambda_{c d}^{T}=\lambda_{a d}^{T}+\lambda_{b c}^{T}$ holds, $\mathcal{L}_{a b c d}$ cannot be part of a basis $B \in \mathbb{M}(T)$. It follows that $\mathbb{M}\left(T_{1}\right) \simeq \mathbb{M}\left(T_{2}\right)$ implies $\mathbb{Q}\left(T_{1}\right)=\mathbb{Q}\left(T_{2}\right)$ where, for any $X$-tree $T, \mathbb{Q}(T)$ is defined by $\mathbb{Q}(T):=\left\{a b\left|c d:\{a, b, c, d\} \in\left(\begin{array}{c}X \\ 4\end{array}\right), T\right|_{\{a, b, c, d\}} \simeq T_{a b \mid c d}\right\}$. However, it has been observed already by H. Colonius and H. Schultze in (Colonius and Schultze, 1977, 1981) that $\mathbb{Q}\left(T_{1}\right)=\mathbb{Q}\left(T_{2}\right)$ holds for any two $X$-trees $T_{1}, T_{2}$ if and only if one has $T_{1} \simeq T_{2}$ (for a more recent account, see (Dress et al., 2011a. Theorem 2.7)) or (Semple and Steel, 2003, Corollary 6.3.8)).

\subsection{The rank of topological lassos}

Now assume that $n \geq 4$ holds and recall that the following three assertions are - according to (Dress et al. 2011b. Theorem 8) - equivalent in this case for any $X$-tree $T=(V, E)$ and any bipartition of $X$ into two disjoint non-empty subsets $A, B$ :

(split-i) The subset $A \vee B$ of $\left(\begin{array}{c}X \\ 2\end{array}\right)$ is a topological lasso for $T$,

(split-ii) $A \vee B$ is a ' $t$-cover' of $T$ (i.e., given any interior vertex $v$ of $T$ and any two edges $e, e^{\prime} \in E$ with $v \in e, e^{\prime}$, there exists some cord in $A \vee B$ with $e, e^{\prime} \in E(x \mid y)$, see (Dress et al. 2011a. Section 7)).

(split-iii) $A \cap\{a, b\} \neq \emptyset \neq B \cap\{a, b\}$ holds for every $T$-cherry $a b^{\text {[iii) }}$

And it was also noted in this context that such bipartitions exist if and only if every $T$-cherry is a proper $T$-cherry and $n \geq 4$ holds.

Here, we want to complement this result as follows:

(iii) When stating this theorem in (Dress et al. 2011b), we omitted to mention that one needs to assume that $n \geq 4$ holds. Indeed, it is simply wrong for $n=3$ for trivial reasons as (split-i) holds for all bipartitions $A, B$ of the leaf set $X$ of an $X$-tree with 3 leaves, but (split-ii) and (split-iii) never holds in this case. Yet, the assumption $n \geq 4$ will always be made here when applying this theorem. 
Theorem 2 Given any $X$-tree $T=(V, E)$, one has $\operatorname{rk}^{T}(\mathcal{L}) \leq|E|-1$ for every bipartite subset $\mathcal{L}$ of $\left(\begin{array}{c}X \\ 2\end{array}\right)$. Furthermore, the following assertions are equivalent for every subset $\mathcal{L}$ of $\left(\begin{array}{l}X \\ 2\end{array}\right)$ that satisfies $\operatorname{rk}^{T}(\mathcal{L}) \leq|E|-1$ :

(i) The rank $\operatorname{rk}^{T}(\mathcal{L})$ of $\mathcal{L}$ coincides with $|E|-1$.

(ii) There exists some cord $x y \in\left(\begin{array}{c}X \\ 2\end{array}\right)$ such that $\mathcal{L} \cup\{x y\}$ is an edge-weight lasso for $T$.

(iii) $\mathcal{L}$ is connected and $\mathcal{L} \cup\{x y\}$ is an edge-weight lasso for $T$ for some cord $x y \in\left(\begin{array}{c}X \\ 2\end{array}\right)$ if and only if $\mathcal{L} \cup\{x y\}$ is not bipartite.

(iv) $\mathcal{L}$ is connected, the closure $[\mathcal{L}]^{T}$ of $\mathcal{L}$ relative to $\mathbb{M}(T)$ coincides with the edge set of the (necessarily unique) complete bipartite graph with vertex set $X$ whose edge set contains $\mathcal{L}$, i.e., $[\mathcal{L}]^{T}$ coincides with the set $A \vee B$ in case the two subsets $A, B$ of $X$ form the (necessarily unique) bipartition of $X$ with $\mathcal{L} \subseteq A \vee B$, and this set forms a 'hyperplane' in $\mathbb{M}(T)$, i.e., a maximal subset of $\left(\begin{array}{c}X \\ 2\end{array}\right)$ of rank smaller than $|E|$.

Proof: Assume that $A$ and $B$ are two subsets of $X$ that form a bipartition of $X$ with $\mathcal{L} \subseteq A \vee B$, and let $\omega_{A \mid B} \in \mathbb{R}^{E}$ denote the map in $\mathbb{R}^{E}$ that maps every interior edge of $T$ onto 0 , every pendant edge $e$ that is incident with some leaf in $A$ onto 1 , and every pendant edge $e$ that is incident with some leaf in $B$ onto -1 . Clearly,

$$
\lambda_{x y}^{T}\left(\omega_{A \mid B}\right)=\left\{\begin{aligned}
+2 & \text { if } x, y \in A, \\
-2 & \text { if } x, y \in B, \\
0 & \text { otherwise }
\end{aligned}\right.
$$

holds for every cord $x y \in\left(\begin{array}{c}X \\ 2\end{array}\right)$. In particular, one has $\lambda_{x y}^{T}\left(\omega_{A \mid B}\right)=0$ for some cord $x y \in\left(\begin{array}{c}X \\ 2\end{array}\right)$ if and only if $x y \in A \vee B$ holds. So, standard matroid theory implies that $\mathrm{rk}^{T}(\mathcal{L}) \leq \mathrm{rk}^{T}(A \vee B) \leq|E|-1$ must hold for every subset $\mathcal{L}$ of a set of the form $A \vee B$ for some bipartition $A, B$ of $X$, that is, for every bipartite subset $\mathcal{L}$ of $\left(\begin{array}{c}X \\ 2\end{array}\right)$ - which is just our first assertion.

(i) $\Leftrightarrow$ (ii): It follows also from standard matroid theory that the rank $\operatorname{rk}^{T}(\mathcal{L})$ of an arbitrary nonempty subset $\mathcal{L}$ of $\left(\begin{array}{c}X \\ 2\end{array}\right)$ with $\operatorname{rk}^{T}(\mathcal{L}) \leq|E|-1$ coincides with $|E|-1$ if and only if there exists some cord $x y \in\left(\begin{array}{c}X \\ 2\end{array}\right)$ such that $\mathcal{L} \cup\{x y\}$ is an edge-weight lasso for $T$.

(i) $\Rightarrow$ (iii): And standard matroid theory implies also that, if $\mathcal{L}$ is any subset of $\left(\begin{array}{l}X \\ 2\end{array}\right)$, one has $\operatorname{rk}^{T}(\mathcal{L})=$ $|E|-1$ if and only if there exists - up to scaling - exactly one non-zero map $\omega \in \mathbb{R}^{E}$ with $\lambda_{a b}^{T}(\omega)=0$ for all $a b \in \mathcal{L}$ and that, in this case, $\mathcal{L} \cup\{x y\}$ is an edge-weight lasso for $T$ for some cord $x y \in\left(\begin{array}{c}X \\ 2\end{array}\right)$ if and only if $\lambda_{x y}^{T}(\omega) \neq 0$ holds for this map $\omega$.

In consequence, if $\mathcal{L} \subseteq\left(\begin{array}{c}X \\ 2\end{array}\right)$ is bipartite and $\operatorname{rk}^{T}(\mathcal{L})=|E|-1$ holds, our observations above imply that there must be a unique bipartition $A, B$ of $X$ with $\mathcal{L} \subseteq A \vee B$ and that, given any cord $x y \in\left(\begin{array}{l}X \\ 2\end{array}\right)$, the union $\mathcal{L} \cup\{x y\}$ is an edge-weight lasso for $T$ if and only if $\lambda_{x y}^{T}\left(\omega_{A \mid B}\right) \neq 0$ and, hence, if and only if $x y \notin A \vee B$ holds. Furthermore, the fact that there is only one bipartition $A, B$ of $X$ with $\mathcal{L} \subseteq A \vee B$ implies that $\mathcal{L}$ must be connected and that, in consequence, $x y \notin A \vee B$ holds if and only if $\mathcal{L} \cup\{x y\}$ is not bipartite.

So, we see that $\mathcal{L} \cup\{x y\}$ is indeed an edge-weight lasso for $T$ if and only if $\mathcal{L} \cup\{x y\}$ is not bipartite, as claimed. 
(iii) $\Rightarrow$ (ii): This is trivial as any connected graph with at least three edges can be extended by a single edge to become a non-bipartite graph.

(i,ii,iii) $\Rightarrow$ (iv): If $\mathcal{L}$ is connected and bipartite, there exists exactly one bipartition $A, B$ of $X$ with $\mathcal{L} \subseteq A \vee B$ and $\mathcal{L} \cup\{x y\}$ bipartite for some cord $x y \in\left(\begin{array}{c}X \\ 2\end{array}\right)$ if and only if $x y \in A \vee B$ holds. So, if in addition also $|E|-1=\mathrm{rk}^{T}(\mathcal{L})$ holds, $[\mathcal{L}]^{T}$ must be a hyperplane and we must have $|E|-1=\mathrm{rk}^{T}(\mathcal{L}) \leq$ $\mathrm{rk}^{T}(A \vee B) \leq|E|-1$. Thus, $\operatorname{rk}^{T}(\mathcal{L})=\mathrm{rk}^{T}(A \vee B)=|E|-1$ as well as

$$
\begin{aligned}
{[\mathcal{L}]^{T} } & =\left\{x y \in\left(\begin{array}{c}
X \\
2
\end{array}\right): \operatorname{rk}^{T}(\mathcal{L} \cup\{x y\}) \leq|E|-1\right\} \\
& =\left\{x y \in\left(\begin{array}{c}
X \\
2
\end{array}\right): \mathcal{L} \cup\{x y\} \text { is bipartite }\right\}=A \vee B
\end{aligned}
$$

as claimed.

(iv) $\Rightarrow$ (i): This is trivial in view of the fact that $\operatorname{rk}^{T}(\mathcal{L})=\mathrm{rk}^{T}\left([\mathcal{L}]^{T}\right)$ holds for every nonempty subset $\mathcal{L}$ of $\left(\begin{array}{l}X \\ 2\end{array}\right)$ and the fact that any maximal subset of $\left(\begin{array}{l}X \\ 2\end{array}\right)$ of rank smaller than $|E|$ must have rank $|E|-1$.

The above theorem has an interesting application regarding topological lassos:

Theorem 3 (i) Given any $X$-tree $T=(V, E)$ and any bipartite subset $\mathcal{L}$ of $\left(\begin{array}{c}X \\ 2\end{array}\right)$ with $\mathrm{rk}^{T}(\mathcal{L})=|E|-1$, the hyperplane $[\mathcal{L}]^{T}$ is a topological lasso for $T$ if and only if every $T$-cherry is a proper $T$-cherry (i.e., if and only if there exists at least one bipartition $A^{\prime}, B^{\prime}$ of $X$ such that $A^{\prime} \vee B^{\prime}$ is a topological lasso for $T$ ). In this case $[\mathcal{L}]^{T} \cup\{x y\}$ must be a strong lasso for $T$ for every cord $x y \in\left(\begin{array}{c}X \\ 2\end{array}\right)$ for which $(X, \mathcal{L} \cup\{x y\})$ is not bipartite, that is, with $x y \notin[\mathcal{L}]^{T}$.

(ii) Conversely, if $\mathcal{L}$ is any topological lasso for $T$ with $\mathrm{rk}^{T}(\mathcal{L})<|E|$, then $\mathcal{L}$ must be bipartite, $\operatorname{rk}^{T}(\mathcal{L})=|E|-1$ must hold, every $T$-cherry must be a proper $T$-cherry, and $\mathcal{L} \cup\{x y\}$ - and not only $[\mathcal{L}]^{T} \cup\{x y\}$ - must be a strong lasso for $T$ for every cord $x y \in\left(\begin{array}{c}X \\ 2\end{array}\right)$ for which $\mathcal{L} \cup\{x y\}$ is not bipartite.

Proof: (i): In view of the equivalence (i) $\Longleftrightarrow$ (iv) of Theorem 2, there must exist a (necessarily unique) bipartition of $X$ into two disjoint subsets $A$ and $B$ such that the hyperplane $[\mathcal{L}]^{T}$ coincides with the set $A \vee B$. Furthermore, this set must also be a topological lasso for $T$ if every $T$-cherry is a proper $T$-cherry: Indeed, in view of the results from (Dress et al. 2011b) quoted above, it suffices to show that, if $a b$ is a proper $T$-cherry and $a \in A$ holds, we must have $b \in B$. Yet, otherwise, we would have $b \in A$ and, therefore, $a b \notin \mathcal{L}$. Since $n \geq 4$, this would allow us to construct yet another non-zero map $\omega_{a b} \in \mathbb{R}^{E}$ with $\lambda_{x y}^{T}\left(\omega_{a b}\right)=0$ for all cords $x y \in \mathcal{L}$ that is not a scalar multiple of $\omega_{A \mid B}$ : Indeed, if the two edges $e_{a}, e_{b} \in E$ containing $a$ and $b$, respectively, share the vertex $v$, there would exist exactly one further edge $e_{a b} \in E$ with $v \in e_{a b}$, and putting $\omega_{a b}\left(e_{a}\right)=\omega_{a b}\left(e_{b}\right):=1, \omega_{a b}\left(e_{a b}\right):=-1$, and $\omega_{a b}(e):=0$ for all other edges $e \in E$ would yield such a map $\omega_{a b}$, as required. So, $[\mathcal{L}]^{T}=A \vee B$ must indeed be a topological lasso for $T$, as claimed. For the 'only if' direction, assume that $[\mathcal{L}]^{T}$ is a topological lasso for $T$. By Theorem 2 ((i) $\Rightarrow$ (iv)) we have $[\mathcal{L}]^{T}=A \vee B$, and from the implication (split-i) $\Rightarrow$ (split-iii) (near the start of Section 5.2) $T$ cannot contain three leaves all of which are adjacent to a common vertex. Thus every $T$-cherry must be a proper $T$-cherry.

(ii) Assume that $\mathcal{L}$ is a topological lasso for $T$ of rank less than $|E|$. Then, there must exist a non-zero map $\omega_{0} \in \mathbb{R}^{E}$ with $\lambda_{x y}\left(\omega_{0}\right)=0$ for all $x y \in \mathcal{L}$. If $\omega_{0}\left(e_{0}\right) \neq 0$ held for some interior edge $e_{0} \in E$, we could find some proper edge-weighting $\omega \in \mathbb{R}_{\geq 0}^{E}$ of $T$ with $\omega\left(e_{0}\right)=\left|\omega_{0}\left(e_{0}\right)\right|$, while $\omega(e)>\omega_{0}(e)$ holds for all edges $e \in E-\left\{e_{0}\right\}$. This, in turn, would imply that the map $\omega^{\prime}:=\omega-\operatorname{sgn}\left(\omega_{0}\left(e_{0}\right)\right) \omega_{0}$ is a 
map in $\mathbb{R}_{\geq 0}^{E}$ that is a non-proper edge-weighting of $T$ for which $\left.D_{(T, \omega)}\right|_{\mathcal{L}}=\left.D_{\left(T, \omega^{\prime}\right)}\right|_{\mathcal{L}}$ holds. In view of the last remark in (Dress et al. 2011b, Subsection 2.2), this would contradict our assumption that $\mathcal{L}$ is a topological lasso for $T$.

Consequently, the support $\operatorname{supp}\left(\omega_{0}\right):=\left\{e \in E: \omega_{0}(e) \neq 0\right\}$ of $\omega_{0}$ must be contained in the set $\left\{e_{x}: x \in X\right\}$ of pendant edges of $T$, implying that $\lambda_{x y}\left(\omega_{0}\right)=\omega_{0}\left(e_{x}\right)+\omega_{0}\left(e_{y}\right)$ must hold for every cord $x y \in\left(\begin{array}{c}X \\ 2\end{array}\right)$. Thus, putting $A:=\left\{x \in X: \omega_{0}\left(e_{x}\right)>0\right\}$ and $B:=\left\{y \in X: \omega_{0}\left(e_{y}\right)<0\right\}$, we see that " $x \in A \Longleftrightarrow y \in B$ " must hold for every cord $x y \in\left(\begin{array}{c}X \\ 2\end{array}\right)$ with $\lambda_{x y}\left(\omega_{0}\right)=0$ and, hence, for all $x y \in \mathcal{L}$.

Thus, as $\mathcal{L}$ must be connected for every topological lasso $\mathcal{L}$ for $T$ in view of (Dress et al., 2011b. Theorem 4), it follows that the pair $A, B$ of subsets of $X$ forms a bipartition of $X$, that $\mathcal{L}$ must be bipartite relative to this partition, and that $A \vee B$ must also be a topological lasso for $T$. Consequently, every $T$-cherry must be a proper $T$-cherry and $\omega_{0}$ must be a (positive) scalar multiple of the map $\omega_{A \mid B} \in \mathbb{R}^{E}$ defined above. In particular, there can be - up to a scaling - only one non-zero map $\omega \in \mathbb{R}^{E}$ with $\lambda_{x y}(\omega)=0$ for all $x y \in \mathcal{L}$ implying that the rank of $\mathcal{L}$ must indeed coincide with $|E|-1$ and that $\mathcal{L} \cup\{x y\}$ must, therefore, be a strong lasso for $T$ for every cord $x y \in\left(\begin{array}{c}X \\ 2\end{array}\right)$ for which $\mathcal{L} \cup\{x y\}$ is not bipartite, i.e. for every cord $x y \in\left(\begin{array}{c}X \\ 2\end{array}\right)-A \vee B$.

Corollary 5.1 Given any $X$-tree $T=(V, E)$, the following assertions are equivalent:

(i) There exists a bipartite subset $\mathcal{L}$ of $\left(\begin{array}{c}X \\ 2\end{array}\right)$ that is a topological lasso for $T$.

(ii) There exists a topological lasso $\mathcal{L}$ for $T$ with $\operatorname{rk}^{T}(\mathcal{L})<|E|$.

$\left(i i^{\prime}\right)$ There exists a topological lasso $\mathcal{L}$ for $T$ with $\operatorname{rk}^{T}(\mathcal{L})=|E|-1$.

(iii) Every $T$-cherry is a proper $T$-cherry.

A simple example to illustrate Corollary 5.1 is presented in Figure 3 .

\section{Concluding comments}

\section{1 $X$-trees $T$ for which $\mathbb{M}(T)$ is a non-binary matroid}

Let us note finally that the matroid $\mathbb{M}(T)$ associated to an $X$-tree $T$ is a non-binary matroid whenever there exist three disjoint cords in $X$ each of which forms a $T$-cherry: Indeed, assume that $x_{1}, x_{2}, x_{3}, x_{4}, x_{5}, x_{6}$ are six distinct elements in $X$ such that each of the three cords $x_{i} x_{i+3}(i=1,2,3)$ forms a $T$-cherry. It is then easy to check that the two subsets $\mathcal{L}_{1}:=\left\{x_{1} x_{2}, x_{2} x_{3}, x_{3} x_{4}, x_{4} x_{5}, x_{5} x_{6}, x_{6} x_{1}\right\}$ and $\mathcal{L}_{2}:=$ $\left\{x_{1} x_{3}, x_{3} x_{4}, x_{4} x_{6}, x_{6} x_{1}\right\}$ of $\left(\begin{array}{c}X \\ 2\end{array}\right)$ are circuits in $\mathbb{M}(T)$ while their symmetric difference

$$
\mathcal{L}:=\mathcal{L}_{1} \triangle \mathcal{L}_{2}=\left\{x_{1} x_{2}, x_{2} x_{3}, x_{3} x_{1}, x_{4} x_{5}, x_{5} x_{6}, x_{6} x_{4}\right\}
$$

is an independent subset of $\left(\begin{array}{c}X \\ 2\end{array}\right)$ in $\mathbb{M}(T)$.

Clearly, this implies that a binary $X$-tree $T$ for which $\mathbb{M}(T)$ is a binary matroid must be a caterpillar tree. More generally, an arbitrary $X$-tree $T$ for which $\mathbb{M}(T)$ is a binary matroid must be either a star tree with at most five leaves or an $X$-tree for which - as in the case of the (binary) caterpillar trees - two interior vertices $u$ and $v$ of $T$ exist for which the path from $u$ to $v$ in $T$ passes every interior vertex of $T$ and all of these except perhaps $u$ and $v$ have degree 3 while the two vertices $u$ and $v$ have degree 3 or 4 . We will show in a separate paper that, conversely, $M(T)$ is a binary matroid whenever this holds. 


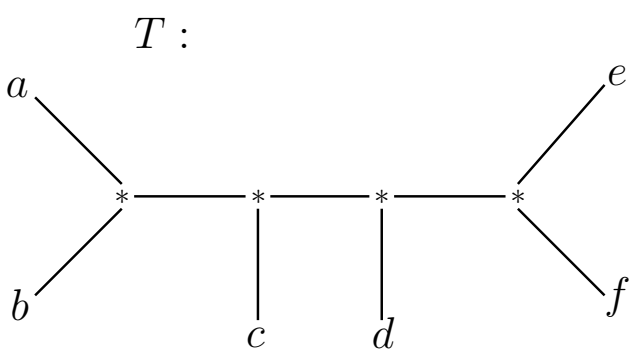

(i)

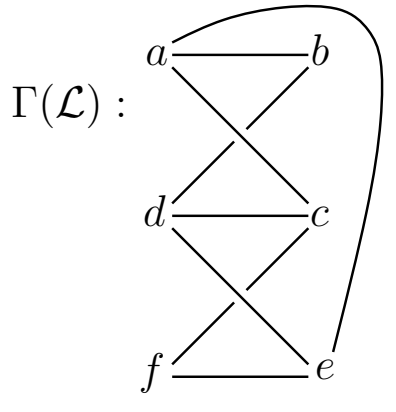

(ii)

Fig. 3:

(i) An $X$-tree $T=(V, E)$ for $X:=\{a, b, c, d, e, f\}$ with only proper $T$-cherries, and (ii) a bipartite topological lasso $\mathcal{L}$ with $\mathrm{rk}^{T}(\mathcal{L})=8(=|E|-1)$. Note that both $T$-cherries $a b$ and ef have a non-empty intersection with both parts $\{a, d, f\}$ and $\{b, c, e\}$ of the bipartition of $X$ induced by $\mathcal{L}$.

\subsection{Minimal strong lassos do not form a matroid}

Although the minimal edge-weight lassos for any $X$-tree form a matroid defined on $\left(\begin{array}{c}X \\ 2\end{array}\right)$, the same is not always true for the minimal strong lassos.

To see this, let $X=\{a, b, c, d, e, f\}$ and consider the sets:

$$
\mathcal{L}_{1}:=\{a b, a c, a d, b c, b d, c d, e f, a e, b e, c e, d e, d f\},
$$

and

$$
\mathcal{L}_{2}:=\{a b, a c, a d, b c, b d, c d, e f, a e, b e, c f, d f\} .
$$

Both $\mathcal{L}_{1}$ and $\mathcal{L}_{2}$ are minimal strong lassos for the $X$-tree that has one interior vertex adjacent to $a, b, c, d$, and a second interior vertex adjacent to $e, f$; however, $\mathcal{L}_{1}$ has one more element than $\mathcal{L}_{2}$.

\subsection{Pointed $x$-covers of binary $X$-trees $T$ that are bases of $\mathbb{M}(T)$}

When $T$ is a binary $X$-tree, some particular bases in $\mathbb{B}(T)$ are easily described: Select any element $x \in X$ and, for each one of the $n-2$ interior vertices $v$ of $T$, consider the three components of the graph obtained from $T$ by deleting $v$. Select an element of $X$ from each of the two components that do not contain $x$, and denote this pair by $y_{v}=y_{v}(x), z_{v}=z_{v}(x)$. Put

$$
\mathcal{L}=\{a x: a \in X-\{x\}\} \cup\left\{y_{v} z_{v}: v \in V-X\right\},
$$

and let $P_{x}(T)$ denote the collection of subsets of $\left(\begin{array}{c}X \\ 2\end{array}\right)$ that can be generated in this way (by the various choices of $y_{v}$ and $z_{v}$ as $v$ varies). 
For example, considering again the quartet $X$-tree $T:=T_{a b \mid c d}$ with its two interior vertices $u$ and $v$ as shown in Figure 1(ii), we may choose $x:=d, y_{u}=y_{v}:=a, z_{u}:=b, z_{v}=c$ and obtain the lasso

$$
\mathcal{L}=\{a d, b d, c d\} \cup\{a b, a c\}
$$

as an element of $P_{d}\left(T_{a b \mid c d}\right)$.

Clearly, $P_{x}(T)$ is a subset of $\mathbb{B}(T)$ for each $x \in X$, since the elements of $P_{x}(T)$ correspond precisely to the so-called 'pointed $x$-covers' of $T$ of cardinality $2 n-3$ and, by Theorem 7 of (Dress et al. 2011b), any pointed $x$-cover $\mathcal{L}$ of a binary $X$-tree is not only an edge-weight, but a strong lasso for that tree.

We note also that, given two distinct elements $x_{1}, x_{2}$ in $X$, a subset $\mathcal{L}$ of $\left(\begin{array}{c}X \\ 2\end{array}\right)$ cannot simultaneously be a pointed $x_{1}$-cover in $P_{x_{1}}(T)$ and a pointed $x_{2}$-cover in $P_{x_{2}}(T)$ unless $T$ is a caterpillar tree with $x_{1}$ and $x_{2}$ at opposite 'ends' of the tree: Indeed, if there exists some $\mathcal{L} \in P_{x_{1}}(T) \cap P_{x_{2}}(T)$, we must have $\left\{y_{v}\left(x_{1}\right) z_{v}\left(x_{1}\right): v \in V-X\right\}=\left\{x_{2} a: a \in X-\left\{x_{1}, x_{2}\right\}\right\}$ implying that the path from $x_{1}$ to $x_{2}$ in $T$ must pass through every interior vertex of $T$.

\section{Acknowledgements}

We thank Geoffrey Whittle for helpful comments and references to some relevant matroid-theory literature. We also thank the two annonymous reviewers. A.D. thanks the CAS and the MPG for financial support; M.S. thanks the Royal Society of New Zealand under its Marsden and James Cook Fellowship scheme.

\section{References}

H. Colonius and H. H. Schultze. Trees constructed from empirical relations. Braunschweiger Berichte aus dem Institut für Psychologie 1, 1977.

H. Colonius and H. H. Schultze. Tree structure from proximity data. Brit. J. Math. Stat. Psych., 34: 167-180, 1981.

A. Dress, K. T. Huber, J. Koolen, V. Moulton, and A. Spillner. Basic phylogenetic combinatorics. Cambridge University Press, 2011a.

A. Dress, K. T. Huber, and M. Steel. 'Lassoing' a phylogenetic tree I: Basic properties, shellings and covers. J. Math. Biol., 65(1):77-105, 2011 b.

A. Guénoche, B. Leclerc, and V. Markarenkov. On the extension a partial metric to a tree metric. Discr. Appl. Math., 276:229-248, 2004.

J. Oxley. Matroid Theory (2nd edition). Oxford University Press, USA, 2011.

M. J. Sanderson, M. M. McMahon, and M. Steel. Phylogenomics with incomplete taxon coverage: the limits to inference. BMC Evol. Biol., 10:155, 2010.

C. Semple and M. Steel. Phylogenetics. Oxford University Press, 2003.

D. A. Welsh. Matroid theory. Academic Press, New York, 1976. 
